\title{
Aider l'apprenti-lecteur en difficulté : analyse comparée des approches didactiques mises en œuvre en France, Au Québec et dans le Bade- Wurtemberg
}

\author{
Caroline Viriot-Goeldel
}

\section{OpenEdition}

\section{Journals}

Édition électronique

URL : https://journals.openedition.org/educationdidactique/307

DOI : 10.4000/educationdidactique.307

ISBN : 978-2-7535-1618-2

ISSN : 2111-4838

\section{Éditeur}

Presses universitaires de Rennes

\section{Édition imprimée}

Date de publication : 1 septembre 2008

Pagination : 81-92

ISBN : 978-2-7535-0750-0

ISSN : 1956-3485

\section{Référence électronique}

Caroline Viriot-Goeldel, « Aider l'apprenti-lecteur en difficulté : analyse comparée des approches didactiques mises en œuvre en France, Au Québec et dans le Bade-Wurtemberg », Éducation et didactique [En ligne], 2-2 I septembre 2008, mis en ligne le 01 septembre 2010, consulté le 16 août 2022. URL : http://journals.openedition.org/educationdidactique/307 ; DOI : https://doi.org/10.4000/ educationdidactique.307 


\title{
AIDER L'APPRENTI-LECTEUR EN DIFFICULTÉ : ANALYSE COMPARÉE DES APPROCHES DIDACTIQUES MISES EN OEUVRES EN FRANCE, AU QUÉBEC ET DANS LE BADE-WURTEMBERG
}

\author{
Caroline Viriot-Goeldel, LISEC EA 2310, IUFM d'Alsace
}

\begin{abstract}
Résumé : En France, 90 \% des enfants qui redoublent l'année de CP n'obtiendront jamais le baccalauréat. C'est dire à la fois combien les premiers apprentissages en lecture sont déterminants et aussi combien il est impératif d'aider efficacement les apprentis-lecteurs à surmonter les premières difficultés qu'ils rencontrent.

Cette recherche traite de l'aide apportée aux apprentis-lecteurs en difficulté en France, au Québec et dans le BadeWurtemberg. Elle compare les approches didactiques mises en œuvre lors de séances de soutien proposées dans le cadre scolaire à des élèves de lère et 2ème année d'école élémentaire. Elle permet non seulement de caractériser différents choix pédagogiques, d'analyser leurs forces et leurs faiblesses, mais aussi d'esquisser les contours de réponses cohérentes aux difficultés d'apprentissage de la lecture.

Mots-clés : Education comparée - Difficultés de lecture - Prévention de l'illettrisme - Littéracie - Didactique de la lecture
\end{abstract}

Caroline Viriot-Goeldel

Diverses études conduites en France situent autour de $15 \%$ le nombre d'élèves éprouvant des difficultés de lecture à l'entrée en $6^{\text {ème }}$ (MEN, 1999; Bonjour \& Gombert, 2004). Ces difficultés relèvent en partie d'une absence d'automaticité des mécanismes d'identification des mots, fréquemment accompagnée de carences prononcées en orthographe ainsi que de faibles capacités phonologiques. À l'entrée en $6^{\text {eme }}$, ce profil concernerait plus de $7 \%$ des élèves, handicapés par une extrême lenteur de ces mécanismes (MEN, 1999). Ces mêmes études soulignent également les difficultés liées à une mauvaise maîtrise des processus de compréhension, y compris parfois chez des élèves maîtrisant le déchiffrage. L'enquête PIRLS (Progress in International Reading literacy Study) dresse un tableau détaillé des compétences en compréhension d'élèves de 10 ans dans 35 pays. Menée en 2001 par l'IEA (International Association for the Evaluation of Educational Achievement), celle-ci fait apparaître, pour ce qui est des élèves français, les difficultés de certains d'entre eux à dépasser la compréhension littérale pour accéder à l'implicite et mener une réflexion personnelle à partir du texte. Ils ne sont en effet que $26 \%$ à posséder les capacités inférentielles et interprétatives qui caractérisent le niveau 3 de compréhension, contre $45 \%$ des élèves anglais. Seuls $9 \%$ des élèves français atteignent le niveau 4 , contre $24 \%$ des élèves anglais (Remond, 2007).
Les études en question soulignent le caractère composite des difficultés de lecture. Elles montrent, s'il en est besoin, qu'il n'existe pas une catégorie homogène de lecteurs en difficulté, un idéal type du mauvais lecteur en quelque sorte, mais une grande diversité des non-performances, ce qui pose de manière aiguë la question de l'aide à apporter aux apprentis-lecteurs en difficulté. La nécessité d'intervenir dès le début de l'apprentissage afin d'éviter que les difficultés ne s'accroissent tout au long d'une scolarité fait désormais consensus. Les modalités mêmes de cette intervention sont en revanche bien moins consensuelles: les dispositifs d'aide et les approches didactiques mis en place dans les différents systèmes scolaires divergent fortement. Notre recherche s'attache à décrire, analyser et comparer l'aide apportée à ces lecteurs en France, dans le Bade-Wurtemberg et au Québec ${ }^{1}$. Par une analyse qualitative, elle confronte les pratiques didactiques observées lors de séances de soutien. Il ne s'agit pas d'évaluer les pratiques observées - nous ne proposerons pas de palmarès de l'aide! -, mais de permettre de mieux comprendre, grâce à la comparaison, la didactique du soutien en lecture mise en oeuvre dans les écoles des trois pays, dans l'espoir de contribuer à nourrir la réflexion théorique sur ce type d'intervention spécifique, et à éclairer l'action auprès des apprentis-lecteurs en difficulté. 


\section{Méthodologie}

\section{Une approche qualitative}

Il s'agissait d'une part d'éviter les écueils liés au déclaratif, et en particulier l'écart entre ce que les maîtres disent faire et ce qu'ils font effectivement, et d'autre part de ne pas enfermer les pratiques dans des cadres trop rigides afin de permettre à de nouvelles données, même totalement inattendues, d'émerger. C'est pourquoi c'est une méthodologie qualitative, permettant d'entrer dans la «boîte noire " des classes, qui a été privilégiée. Les données relatives aux approches didactiques mises en œuvre pour aider les apprentis-lecteurs en difficulté ont été collectées au moyen d'une « observation participante » (Bryman, 2000) de séances de soutien.

\section{Echantillon}

Le terrain de cette recherche est constitué de vingtquatre classes réparties dans vingt écoles élémentaires françaises, allemandes et québécoises. Les écoles ont été choisies pour représenter des milieux socioéconomiques variés et équivalents dans les trois pays. $\mathrm{Au}$ sein de ces établissements, les apprentis-lecteurs en difficulté bénéficient de dispositifs d'aide de toutes sortes : aide apportée par le maître en classe; aide apportée par un autre enseignant ou un enseignant spécialisé; dans ou hors de la classe; pendant ou en dehors du temps scolaire. Vingt-quatre séances de soutien ont été retenues dans différents dispositifs ${ }^{2}$ de l'échantillon et ont fait l'objet d'une observation participante, soit huit dans chacun des trois pays ${ }^{3}$. Elles ont été choisies au hasard à différents moments de l'année.

\section{Recueil et traitement des données}

Afin de recueillir les contenus de ces de ces vingtquatre séances, une grille d'observation a été constituée. Elle permet un codage des différentes activités proposées par l'enseignant organisé autour de cinq domaines spécifiques que constituent l'expression orale, la conscience phonologique, l'identification des mots, la compréhension et l'écriture. Ce codage a été accompagné d'une prise de note en temps réel, mentionnant de manière plus détaillée les supports utilisés, les performances des élèves et les difficultés rencontrées.

La grille ainsi complétée offre un panorama des compétences traitées dans les séances des trois pays : 


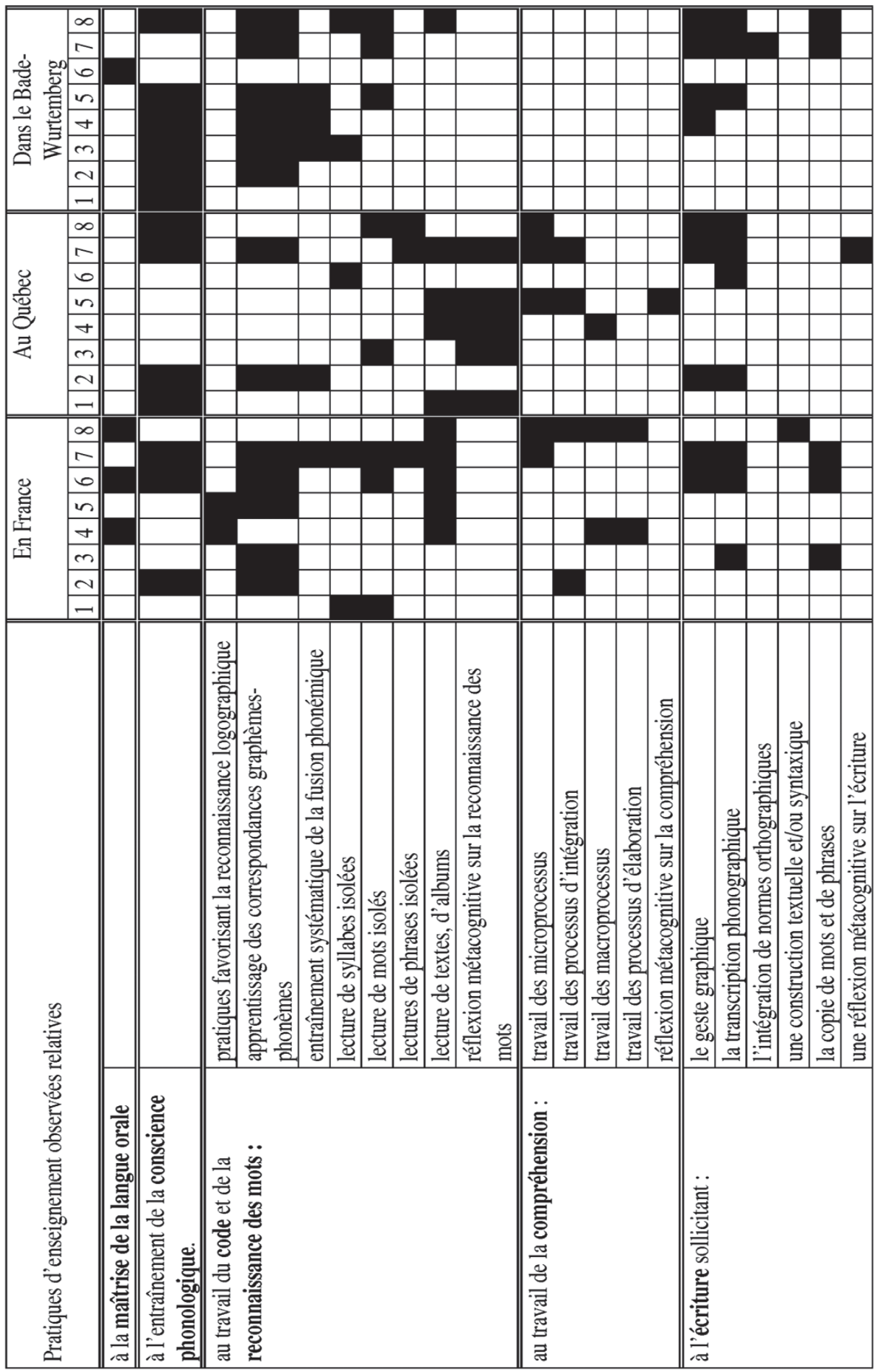

Synthèse des contenus didactiques des séances observées dans les trois pays 
Larticle ne rendra compte que partiellement de l'analyse qui peut être faite de ce tableau. Celle-ci prend notamment appui sur le modèle de la lecture exposé par Frith (1985), sur les travaux de Gombert relatifs à la métaphonologie (1990), sur ceux de Giasson concernant la compréhension (2008), ainsi que sur les recherches de Ferreiro et Besse consacrées à l'écriture (Feirreiro \& Gomez Palacio, 1988; Besse, 2001).

Si ces pratiques observées valent par leur exemplarité, elles ne peuvent toutefois prétendre à une représentativité statistique de l'aide aux élèves en difficulté dans les trois pays, du fait des modalités de leur recueil. C'est pourquoi nous avons procédé à une triangulation ${ }^{4}$ avec deux autres types de sources : les instructions et publications officielles d'une part, et la littérature didactique consacrée à l'aide à l'apprentilecteur en difficulté d'autre part. Cette méthode nous a permis d'établir s'il s'agissait de pratiques isolées ou au contraire de pratiques s'inscrivant dans des tendances nationales.

\section{Echantillon québécois : le choix de l'enseignement stratégique}

Les séances de soutien observées au Québec alternent des situations de lecture et de structuration du code. Les orthopédagogues y mettent notamment l'accent sur l'enseignement des "stratégies ». Les stratégies d'identification des mots, parfois modélisées par des pictogrammes affichés, sont ainsi sollicitées durant quatre de ces séances de soutien. Concrètement, on distingue par exemple « je reconnais le mot " (récupération en mémoire d'un mot déjà connu), « je regarde autour du mot » (utilisation du contexte), « je découpe le mot en lettres et en syllabes » (mise en œuvre des correspondances grapho-phonémiques pour identifier le mot), « je reconnais certains mots costumés » (utilisation de la morphologie). Par ailleurs, on cherche également à développer des stratégies de compréhension ou d'écriture. Durant les séances observées, l'approche stratégique est généralement accompagnée d'une réflexion métacognitive.

Lexamen d'autres sources confirme que les pratiques d'enseignement stratégique observées dans l'échantillon sont loin de constituer des pratiques isolées. "Stratégies de lecture », "stratégies d'écriture », « stratégies de communication orale », « stra- tégies liées à l'appréciation d'œuvres littéraires » : les compétences du programme québécois de français langue d'enseignement se déclinent invariablement en termes de stratégies, aussi bien pour l'enseignement de la lecture que pour le soutien des élèves en difficulté (MEQ, 2001; 2003). Les manuels de lecture, qui font une large part à l'approche stratégique, jouent un rôle de courroie de transmission entre les programmes et les enseignants. Cela s'explique notamment par le fait que l'accréditation d'un manuel dépend de sa conformité aux contenus, aux orientations et aux exigences pédagogiques et didactiques des programmes.

Ce choix didactique de l'enseignement stratégique, impulsé au niveau ministériel, s'appuie sur une abondante littérature québécoise largement diffusée auprès des enseignants. Pour un enseignement stratégique (Tardif, 1992) pose les bases d'un modèle pédagogique dans lequel la connaissance des stratégies et de leur utilisation ainsi qu'une connaissance de son propre fonctionnement permet à l'élève un meilleur transfert des apprentissages. Lapproche stratégique a été reprise dans de nombreux ouvrages à destination des enseignants, comme ceux de Giasson, pour qui l'apprentilecteur est un " apprenti stratège en lecture », et le lecteur expert un « lecteur qui utilise les stratégies de lecture » (1995). Van Grunderbeeck distingue quant à elle trois types de stratégies de lecture :

- les stratégies d'identification des mots;

- les stratégies de compréhension;

- les stratégies d'adaptation de la lecture à la situation et au but poursuivi (Van Grunderbeeck, 1994).

Lauteur établit des profils de lecteurs en difficulté en fonction des stratégies qu'ils utilisent et propose des pistes d'intervention adaptées à chaque profil (ibid.).

On observe ainsi une étroite congruence entre les pratiques observées dans l'échantillon, les instructions officielles et la littérature didactique à destination des enseignants.

\section{Fondements de l'enseignement stratégique}

Si enseignants et programmes mentionnent différentes stratégies de lecture, nul ne précise en 
revanche en quoi les processus et mécanismes en question constituent des stratégies, ni ce qu'est une stratégie. Louvrage de Tardif permet toutefois d'appréhender la spécificité de l'enseignement stratégique (1992). Il s'appuie sur une conception cognitiviste des apprentissages, considérés comme des activités de traitement de l'information au cours d'un processus actif et constructif. Tardif met l'accent sur l'aspect intentionnel de la stratégie: ces stratégies se distinguent de simples processus et mécanismes dans la mesure où elles peuvent être définies comme l'organisation planifiée par le sujet de méthodes, techniques et moyens, en vue d'atteindre un objectif. Il souligne également le statut de connaissance des stratégies. L'enseignement stratégique vise à amener l'élève à développer non seulement le «quoi » (les connaissances déclaratives) et le « comment» (les connaissances procédurales), mais aussi le «pourquoi » et le «quand (les connaissances conditionnelles). Ce sont ces connaissances conditionnelles qui permettent le transfert des apprentissages d'un contexte à un autre. Enfin, il importe que l'élève soit conscient des stratégies disponibles et efficaces pour réaliser la tâche. Dans la réalisation d'une tâche, en plus de connaître les stratégies les plus adéquates qui lui permettent d'accomplir ce qui est demandé, l'élève doit être en mesure de les gérer activement. Il doit pouvoir les contrôler, c'est-à-dire gérer leur application à toutes les étapes de la réalisation et les évaluer. L'enseignement stratégique vise ainsi à développer la métacognition, c'est-à-dire la connaissance et le contrôle qu'une personne a sur elle-même et sur ses stratégies cognitives (ibid., p. 59).

\section{Forces et limites de l'enseignement stratégique}

Lutilisation de l'enseignement stratégique comme voie privilégiée de remédiation auprès des apprentislecteurs en difficulté appelle plusieurs remarques.

L'enseignement stratégique vise à amener l'apprenti-lecteur à mettre des stratégies en œuvre à bon escient, dans le bon contexte et au bon moment, ainsi qu'à les contrôler pendant la lecture. Plusieurs recherches suggèrent la pertinence de ce type d'approches qui permettent de développer un contrôle métacognitif, car c'est bien souvent la qualité de ce contrôle qui différencie les élèves performants de ceux qui éprouvent des difficultés d'apprentissage.
Linefficacité des stratégies cognitives des élèves en difficulté a en effet été confirmée par de nombreuses études. Ces dernières ont également montré les effets positifs sur les apprentissages d'un entraînement métacognitif (voir Gersten, Fuchs, Williams, $\&$ Baker, 2001, pour une méta-analyse concernant la compréhension en lecture). C'est pourquoi ce choix d'une approche centrée sur les forces cognitives de l'élève semble constituer une piste d'intervention pertinente.

Il est toutefois apparu que l'enseignement stratégique échouait parfois à doter l'élève de ce contrôle métacognitif qu'il est censé lui apporter. Une des limites observée concerne en effet le passage des connaissances déclaratives sur les stratégies à la mise en œuvre de ces mêmes stratégies. L'apprenti-lecteur peut avoir appris plusieurs stratégies permettant de reconnaître un mot, mais être incapable de les activer dans une situation de lecture, faute d'une maîtrise suffisante des connaissances procédurales (défaut d'automatisation du décodage par exemple) ou conditionnelles (quand faut-il découper des mots en syllabes?). À l'enseignant de négocier ce délicat passage d'un type de connaissances aux deux autres, permettant la mise en œuvre et le transfert des stratégies, et de veiller à ce que les élèves ne se cantonnent pas à la simple récitation d'une palette de stratégies que nous avons pu observer à plusieurs reprises. Alors seulement, l'apprenti-lecteur sera en mesure de "se dépanner » pendant la lecture et de devenir un lecteur autonome.

Lanalyse a mis au jour d'autres limites de l'enseignement stratégique. Les stratégies de lecture sont d'une part, pour certaines enseignantes interrogées, limitées aux stratégies d'identification des mots. Les traditionnelles «questions de compréhension» demeurent encore souvent le moyen privilégié d'aborder la compréhension. Certaines de ces stratégies d'identification des mots peuvent d'autre part susciter quelques réserves. Lapproche observée sur le terrain encourage des mécanismes tels que l'appui sur le contexte sémantique pour deviner un mot que l'on ne décode pas, ou que l'on décode partiellement. Or on sait maintenant que ce processus est un processus compensatoire utilisé essentiellement par les mauvais lecteurs pour pallier un décodage déficient (Stanovich, 1980). Certains auteurs pensent que cet appui est bénéfique (Goigoux, 2001), tandis que d'autres estiment qu'il ne doit pas être encouragé 
(Chardon \& Baillé, 2004). De la même façon, une des stratégies largement utilisée en classe, ainsi qu'en orthopédagogie fait appel à la reconnaissance logographique. Or il n'existe pas aujourd'hui de consensus clair sur le rôle de la reconnaissance logographique dans l'acquisition de la lecture.

Enfin, le choix ministériel d'imposer l'enseignement stratégique en lecture via les programmes de l'école québécoise et leurs divers documents d'accompagnement fait ainsi apparaître cette approche comme une "méthode officielle ", et la tentation peut être grande de l'appliquer à tous et en toute circonstance, au détriment d'une remédiation plus ciblée.

\section{Echantillon du Bade-Wurtemberg : une "boite à outils » pour surmonter des déficits instrumentaux}

A l'opposé de la métacognition et du contrôle des processus mis en œuvre dans l'échantillon québécois, les interventions de l'échantillon allemand reposent essentiellement sur des exercices de structuration organisés par des progressions structurées. Les enseignants mettent en œuvre une gamme de techniques spécifiques visant à travailler séparément différentes habiletés à l'aide d'une large palette d'outils. Dès lors, venir en aide à l'apprenti-lecteur en difficulté revient à lui proposer l'outil approprié.

Toutes les séances de l'échantillon allemand témoignent d'une approche strictement phonique centrée sur le décodage au moyen d'une démarche essentiellement synthétique, où l'analyse est réservée aux tâches d'écriture. Cette approche est caractérisée par une progression régulière des unités les plus simples vers les plus complexes à l'aide d'exercices de structuration. Les tâches morcelées sont ainsi privilégiées au détriment de situations de lecture signifiantes; lecture et écriture de lettres et de syllabes sont entraînées par des exercices répétitifs et au moyen d'une palette d'outils spécifiques (Silbenhaus, Silbenteppich...).

Outre le poids de l'entraînement phonologique et des exercices de discrimination visuelle, la démarche est également caractérisée par une approche sensorimotrice. De nombreux exercices moteurs plus ou moins en lien avec l'écrit sont proposés. Mentionnons notamment l'utilisation de lettres tactiles ou encore l'usage systématique d'une méthode gestuelle équiva- lant à la méthode Borel-Maisonny (Gebärdesprache, Dummer-Smoch, 2000). Plusieurs de ces exercices font appel à une perception visuo-haptique. Les séances observées ne mettent en revanche en œuvre aucun travail de compréhension et la littérature de jeunesse n’y est pas utilisée.

D’une manière générale, les huit séances analysées présentent une homogénéité très forte. Les méthodes et outils qui y sont mis en œuvre sont connus et partagés par l'ensemble des enseignants spécialisés de l'échantillon, voire des enseignants ordinaires qui déclarent également parfois les utiliser. L'homogénéité constatée dans l'ensemble des huit séances semble être le fruit d'instructions officielles détaillées mentionnant ces approches (K.u.U., 1994), d'autres publications ministérielles didactiques (K.u.U., 1997; 1999), ainsi que de publications didactiques utilisées par les enseignants de l'échantillon et diffusant ces approches et ces outils auprès des praticiens (Breuer \& Weuffen, 2006; DummerSmoch \& Hacketal, 2007; Michel, 2000). L'analyse a en effet montré combien les pratiques observées dans l'échantillon du Bade-Wurtemberg, loin de constituer des pratiques isolées, s'inscrivaient dans une longue tradition de remédiation en Allemagne.

\section{Fondements de l'approche allemande}

Sans doute les choix effectués sont-ils en partie induits par les caractéristiques de la langue allemande. L'allemand présente en effet des correspondances graphèmes-phonèmes plutôt régulières, se prêtant ainsi plus facilement à des approches privilégiant l'aspect phonique que des langues irrégulières (Wimmer, 1993, p. 98). Cela étant, centrer l'intervention auprès des apprentis-lecteurs en difficulté sur l'identification des mots, segmenter à l'extrême les habiletés de décodage, et par conséquent, les entraîner en dehors de tout contexte sémantique, correspond à l'approche diffusée par plusieurs publications officielles. L'une d'entre elles rappelle par exemple que « les plus grands problèmes des élèves en difficulté dans l'apprentissage de la lecture-écriture se situent en dessous du niveau du mot» (Tacke, 1998). En effet, les élèves en difficulté dans l'apprentissage de la lecture-écriture

« ne sont pas en mesure de segmenter les mots en sons isolés (...), il leur est difficile de mémoriser la relation 
entre la lettre et le son (correspondances graphèmes phonèmes), ils ne parviennent pas à fusionner les lettres (synthèse) et n'arrivent pas à articuler les différentes unités en mots en lisant» (ibid., p. 8-9).

Par conséquent, l'intervention auprès des apprentis-lecteurs en difficulté doit reposer sur des exercices systématiques répétés au niveau du mot et de ses constituants, lettres et syllabes, à l'aide notamment d'outils semblables à ceux observés dans l'échantillon (K.u.U, 1994, 1997). Un texte mentionne même que les élèves en difficulté « ne devraient passer à la lecture normale seulement lorsqu'ils savent lire couramment des syllabes » (K.u.U., 1994, p. 15).

Quant à l'approche sensori-motrice, les textes et la littérature officielle en font un incontournable de l'enseignement de la lecture en général aussi bien que de l'intervention spécifique auprès des apprentis-lecteurs en difficulté (K.u.U. 1994; 1997). Ils proposent aux praticiens pléthore d'outils et de méthodes pour la mettre en œuvre, dont la méthode gestuelle qui s'est « imposée comme la « voie royale » de la prévention des difficultés d'apprentissage de la lecture-écriture » en Allemagne (Bartnizky et Christiani, 1993, p. 104). Plusieurs ouvrages consacrés aux difficultés de lecture mettent également l'accent sur l'importance de la perception visuelle, auditive, kinesthésique et rythmique dans l'apprentissage de la lecture. Ils proposent des démarches de soutien fondées sur l'entraînement des différentes fonctions perceptives : entraînement de la perception phonémique, visuelle... (Breuer et Weuffen, 2006; Michel, 2000 ou Rinderle, 2000). Cette approche puise vraisemblablement sa source dans la tradition ancienne d'approches américaines des années 1920, comme la «VAKT » de Fernald comportant des voies d'entrées Visuelle, Auditive, Kinesthésique et Tactile, ou encore l'approche d'Orton et Gilligham. Certains de ces exercices sensori-moteurs, notamment tactiles, comme les dominos tactiles et le dé en relief utilisés dans les séances observées, ou encore la perception sensori-motrice des lettres mise en cuvre dans l'échantillon, semblent quant à eux tout droit sortis des ouvrages de Maria Montessori.

\section{Forces et limites de cette « boîte à outils "}

Les interventions observées auprès des élèves en difficultés de l'échantillon allemand offrent une place de choix à l'enseignement du décodage, déployant des techniques et des outils variés dans les domaines de la conscience phonologique, de la mémorisation des correspondances grapho-phonémiques et de la fusion phonémique. Cette approche présente l'avantage de favoriser l'automatisation des processus d'identification des mots, or on connaît désormais l'importance de l'automaticité de ces processus pour la compréhension en lecture.

Ces habiletés isolées ne sont toutefois pas réinvesties dans des situations de lecture afin d'en favoriser le transfert, et on constate dans les séances observées comme dans les textes officiels et dans la littérature didactique l'absence de travail sur le sens.

Mentionnons à ce sujet que les difficultés d'apprentissage de la lecture ont en Allemagne longtemps été appréhendées exclusivement sous l'angle de la dyslexie. Certains dispositifs d'aide étaient d'ailleurs à l'origine réservés aux enfants dyslexiques, dont les difficultés touchaient essentiellement au domaine de la reconnaissance des mots. Ce qui explique également, dans une certaine mesure, les approches sensori-motrices, elles aussi largement inspirées d'une tradition d'intervention auprès d'élèves dyslexiques. Ces approches sont, du reste, aujourd'hui largement remises en question. La recherche a en effet montré la faible efficacité de certains de ces exercices exclusivement sensori-moteurs, fondés sur une conception dépassée de la dyslexie (voir Van Hout et Estienne, 2001 pour une synthèse). Plusieurs études empiriques allemandes sur les effets de ces pratiques sur l'apprentissage de la lecture et le soutien en lecture ne révèlent elles aussi que des résultats décevants (Brezing, 2000, p. 9; Michel (dir.), 2000, p. 11). Il en serait de même pour les exercices d'orientation visuospatiale observés dans plusieurs séances et, dans une moindre mesure, pour les exercices de latéralisation. Ces exercices, encore largement répandus, pourraient tout au plus favoriser la détente et la concentration avant ou pendant une séance de soutien.

Certains chercheurs soulignent toutefois qu'il importe de distinguer l'entraînement isolé de fonctions telles que la perception tactile ou visuelle de leur entraînement « intégré », en lien avec le langage (Michel (dir.), 2000). Scansion des syllabes, entraînement de la fusion phonémique associant prononciation et écriture, méthode gestuelle et autres exer- 
cices associant la parole et le mouvement seraient ainsi susceptibles d'aider les élèves en difficulté. Pour d'autres, c'est plus spécifiquement un entraînement phonologique associé à une exploration visuohaptique et haptique des lettres qui serait «plus efficace que la simple exploration visuelle » (Gentaz et al., 2004, p. 29). Certains exercices multi-sensoriels mis en œuvre dans l'échantillon allemand seraient ainsi caducs, alors que des recherches récentes confèrent à d'autres un nouvel intérêt.

Echantillon français : recherche d'équilibre et pluralisme méthodologique

D’une manière générale, les séances observées dans les dispositifs d'aide français témoignent d'une recherche d'équilibre entre les différentes dimensions de la lecture ainsi que d'un souci de mettre en place des situations de lecture signifiantes. Pour cela, les séances observées dans l'échantillon français privilégient souvent la lecture de textes entiers. Certaines d'entre elles proposent parfois conjointement ou successivement un travail du code. Pour faire lire ces textes à des élèves en difficulté, les enseignants ont recours à divers types d'étayages qu'ils mettent en place au sein de dispositifs didactiques parfois complexes.

Maîtres de soutien et enseignants spécialisés mettent en œuvre une pluralité de méthodes. Pour résoudre des difficultés spécifiques liées au décodage, un enseignant recourt ponctuellement à la méthode Borel-Maisonny tandis qu'un autre utilise en appoint l'alphabet de La planète des Alphas (Dubois et Huguenin, 2000). D'autres enfin guident simplement pas à pas le décodage des élèves. Les pratiques d'écritures sont également très variées : écriture de mots à l'aide d'étiquettes lettres ou syllabes, écriture collective de mots, copie différée, dictée à l'adulte... La même variété caractérise les temps consacrés à la compréhension, si ce n'est que deux séances comportent un débat autour d'albums, visant à la construction collective de la compréhension d'un texte par le biais du dialogue. Menant les élèves vers une compréhension inférentielle, voire interprétative, elles sont les seules des vingt-quatre séances observées à poser les bases d'une lecture littéraire.
Les séances observées dans l'échantillon français témoignent ainsi d'une grande diversité de pratiques, que l'on peut difficilement prétendre caractériser. Contrairement aux échantillons allemand et québécois, aucun choix méthodologique commun à l'ensemble des séances n'apparaît. Chaque enseignant y fait plein usage de sa " liberté pédagogique » sans que n'y apparaisse de culture pédagogique commune en matière d'aide aux apprentis-lecteurs en difficulté. La consultation des textes officiels révèle que contrairement à ce qui se passe dans les deux autres pays, les instructions officielles ont longtemps "refus(é) toute forme de technicité pédagogique » (Gillig, 1998, p. 99). Et si cette tradition a pris fin avec la publication récente de la série des documents d'accompagnement Lire au CP (MEN, 2003; 2004), lesdits documents ne prônent pas d'approche unique, mais au contraire, une adaptation des réponses aux caractéristiques des élèves.

Quant à l'examen de la littérature didactique française consacrée à l'aide aux apprentis-lecteurs en difficulté, il s'est avéré délicat. Alors que les programmes québécois et les publications officielles du BadeWurtemberg contiennent des références explicites à la fois à une littérature scientifique et à de nombreux ouvrages à destination des enseignants, il n'y a pas de références théoriques explicites dans les textes officiels français. C'est pourquoi il est difficile de pointer la littérature faisant référence en la matière. Lors des entretiens et des observations de séances, il arrivait régulièrement que des enseignants québécois ou allemands évoquent et nous montrent diverses publications didactiques. Rien de tel non plus dans l'échantillon français. Etevé et Gambart ainsi que Goigoux (Goigoux, 1997) soulignent par ailleurs la faiblesse des lectures professionnelles des enseignants français. Faute de références explicites, la triangulation avec des ouvrages didactiques est ainsi bien malaisée dans le cas français. Seules quelques pratiques observées çà et là évoquent l'un ou l'autre courant scientifique ou didactique. C'est le cas notamment des débats interprétatifs. Sans doute impulsé par les travaux de Tauveron (Tauveron (dir.), 2002) et relayé par les textes officiels du cycle 3 (MEN, 2002), ce dispositif didactique n'est toutefois pas spécifiquement conçu à destination des apprentis-lecteurs en difficulté.

À la lumière des exemples québécois et allemand et du rôle qu'y jouent les textes officiels et 
la littérature didactique dans l'uniformité des pratiques d'intervention, on comprend mieux dès lors pourquoi les séances observées dans notre échantillon français ne présentent pas l'homogénéité des pratiques didactiques observées lors des séances québécoises et allemandes de l'échantillon. Leur pluralité d'approche semble même induite par des textes officiels dans un premier temps peu prescriptifs en matière didactique, et dans un deuxième temps prescripteurs d'une approche individualisée qui témoigne de la conception de la lecture comme un acte complexe et multidimensionnel dont toutes les composantes doivent être travaillées de manière systémique.

\section{Forces et limites}

Dans ces conditions, il paraît difficile d'évoquer les forces et les limites d'une approche aussi malaisée à caractériser et à la représentativité incertaine. On peut toutefois s'autoriser quelques remarques ponctuelles sur les séances d'aide mises en œuvre. Lavantage de l'approche française est qu'elle est, aussi bien du point de vue du curriculum prescrit que du curriculum réel, méthodologiquement plus ouverte que celles observées au Québec et dans le Bade-Wurtemberg. On n'y observe pas de démarche privilégiant un aspect au détriment des autres, et appliquée à tous sans distinction.

Mais il y a le revers de la médaille: l'analyse a montré quel était parfois le prix à payer pour assurer l'équilibre entre les différents domaines. Proposer un texte entier à des élèves de $\mathrm{CP}$ en difficulté dans le domaine de la reconnaissance des mots nécessite un étayage adapté. À plusieurs reprises cependant, les étayages proposés se sont avérés insuffisants, quand ils n'induisaient pas d'effets négatifs. La reconnaissance de mots par la voie indirecte a parfois cédé le pas à l'utilisation de processus compensatoires, tels que la récitation d'un texte connu par cœur, le devinement, ou la reconnaissance logographique des silhouettes de mots-étiquettes. À vouloir poursuivre simultanément plusieurs objectifs, il arrive que certains dispositifs didactiques complexes observés manquent leurs objectifs, dévoilant les difficultés d'un tel équilibre.
Enfin, si Lire au CP préconise une approche individualisée de l'élève en difficulté, les séances collectives d'aide observées hors de la classe proposent à tous les mêmes exercices.

\section{Regards croisés}

Lanalyse approfondie de ces données a permis de mettre au jour trois approches didactiques distinctes à destination des apprentis-lecteurs en difficulté. Elle a révélé que l'échantillon québécois se caractérisait par un enseignement stratégique de la lecture ménageant un espace aux processus métacognitifs. Les programmes du Bade-Wurtemberg de 1994 et la littérature allemande en la matière ont favorisé une remédiation fondée sur un entraînement intensif du décodage par une extrême segmentation des tâches et au moyen d'une approche sensori-motrice forte d'une profusion d'outils. L'approche française, quant à elle, s'est moins aisément laissée caractériser, si ce n'est par la recherche d'équilibre entre les différentes dimensions de la lecture au moyen d'une pluralité de méthodes.

L'analyse conduite a montré, pour les cas québécois et allemand au moins, combien il ne s'agissait pas de choix isolés, mais d'approches impulsées par un contexte national via les débats sur les méthodes de lecture, les programmes de l'école primaire, les publications officielles et la littérature à destination des enseignants. Au-delà de la lecture, elles témoignent même de différentes conceptions de l'apprentissage et de l'enseignement. En cela, nous sommes bien en présence de tendances nationales d'une didactique du soutien en lecture que cette recherche a permis de mettre en évidence. Au niveau local, enseignants et enseignants spécialisés des écoles s'approprient ainsi ces approches pour construire leurs séances de soutien. Cela est moins évident en France, où le contexte national n'a par le passé jamais vraiment induit d'approches spécifiques, et préconise depuis peu une individualisation des démarches de soutien.

Certes, au cours de l'analyse, les approches allemandes et québécoises ont pu paraître plus systématiques et mieux organisées. Notre étude a toutefois mis au jour certaines forces, mais aussi plusieurs limites de chacune de ces trois approches. On est ainsi face à un phénomène complexe qui ne semble pas souf- 
frir de pensée unique. Loin d'offrir ainsi un modèle à imiter, la comparaison ouvre alors des pistes de réflexion.

De façon générale, notre étude a également montré que dans les trois systèmes décrits, les approches ont souvent été appliquées à tous les apprentis-lecteurs en difficulté, sans que soit opérée de distinction en fonction du profil des élèves. Aussi, un effort d'individualisation et d'adaptation de l'aide semble partout s'imposer, y compris dans le cas de l'échantillon français où il est pourtant préconisé par les documents Lire au CP (1) et (2), mais non encore réalisé dans les faits.

Enfin, en l'absence de données chiffrées sur la progression des élèves, nous ne pouvons porter d'appréciation sur l'efficacité de ces différentes approches. Leur évaluation constitue d'autres perspectives de recherche à explorer. 


\section{NOTES}

1. Rappelons que le Land du Bade-Wurtemberg et la Province de Québec sont tous deux souverains en matière d'éducation, c'est pourquoi, on ne peut effectuer une comparaison qu'avec le Bade-Wurtemberg et le Québec, à défaut de pouvoir le faire avec la RFA et le Québec.

2. Les séances ont été observées dans les dispositifs suivants :

- regroupement d'adaptation (France)

- $\quad$ cours de soutien dans le cadre scolaire (France)

- séances d'orthopédagogie (Québec)

- $\quad$ entraînement à la perception Wahrnehmungstraining (Bade-Wurtemberg)

- $\quad$ école de la lecture Leseschule (Bade-Wurtemberg)

- $\quad$ dispositif LRS (Lese- Rechtschreib-Schwächekurs) (Bade-Wurtemberg).

3. Sept séances adressées à des élèves de $1^{\text {ère }}$ année d'école primaire et une séance adressée à des élèves de $2^{\text {ème }}$ année ont été observées dans chaque pays.

4. La triangulation consiste à faire corroborer les résultats en ayant recours à plusieurs sources de données. Si l'on s'en tient à la distinction classique, elle se réalise à partir de différentes sources de données (personnes, périodes, lieux), de différents types de données (qualitatives, quantitatives...), de différentes méthodes (observation, entretiens, documentation) ou de différents chercheurs (Hubermann et Miles, 2003).

\section{BIBIOGRAPHIE}

Bartnizky, H. \& Christiani, R. (1993). Lese- und Schreibschwierigkeiten vorbeugen und überwinden. Frankfurt/M : Cornelsen Verlag.

Besse, J.-M. (2001). Laccès au principe phonographique : ce que montrent les écritures approchées. In G. Chauveau (Ed.), Comprendre l'apprenti-lecteur (pp. 130-158). Paris : Retz.

Bonjour, E. \& Gombert, J.-E. (2004). Profils de lecteurs à l'entrée en sixième. L'Orientation Scolaire et Professionnelle, 33 (1), 69-101.

Breuer, H. \& Weuffen, M. (2006 pour la $4^{\text {ème }}$ édition). Lernschwierigkeiten am Schulanfang. Weinheim : Beltz-Verlag.

Brezing, H. (2000). Hauptwege, Nebenwege und Irrwege der Lese-Rechtschreibfördernug. In H.-J. MICHEL (Ed.), FRESCH. Grundlagen, Diagnosemöglichkeiten, praktische Übungen zum Thema LRS (pp.4-13). Lichtenau : AOL Verlag.

Bryman, A. (2000). Quantitativisme et qualitativisme : un faux débat? In J.-M. Berthelot (Ed.), Sociologie. Epistémologie d'une discipline. Textes fondamentaux (pp. 209-220). Bruxelles : De Boeck.

Chardon, S. \& Baille, J. (2004). Quels entraînements à la lecture proposer aux élèves de l'école élémentaire? In E. Gentaz \& P. Dessus (Eds.), Comprendre les apprentissages (pp. 33-48). Paris: Dunod.

Dummer-Smoch, L. (2000). Laute-Silben-Wörter / Übungsbuch zum Lesenlernen mit Lautgebärden. Kiel : Veris Verlag.

Dummer-Smoch, L. \& Hacketal, R. (2007 pour la $7^{e}$ édition). Handbuch zum Kieler Leseaufbau. Kiel : Veris Verlag.

Feirrero, E. \& Gomez Palacio, M. (1988). Lire-écrire à l'école. Comment s’y apprennent-ils? Lyon : CRDP.

Frith, U. (1985). Beneath the surface of developmental dyslexia. In K. Patterson, J. Marshall, \& M. Coltheart (Eds.), Surface dyslexia (pp. 301-330). London : Erlbaum.

Gersten, R.; Fuchs, L. ; Williams, J., \& Baker, S. (2001). Teaching Reading Comprehension Strategies to Students with Learning Disabilities : A Review of Research. Review of Educational Research, 10 (2), 279-320.

Giasson, J. (2008 pour la $3^{e}$ édition). La compréhension en lecture. Bruxelles : De Boeck. 
Giasson, J. (1995). La lecture. De la théorie à la pratique. Boucherville : Gaëtan Morin.

Gillig, J.-M. (1998). L'aide aux enfants en difficulté à l'école. Paris : Dunod.

Goigoux, R. (1997). Les élèves en grande difficulté de lecture et les enseignements adaptés. Suresne : CNEFEI.

Goigoux, R. (2001). De l'importance du contexte littéral au début de l'apprentissage de la lecture. In G.

Chauveau (Ed.), Comprendre l'apprenti-lecteur (pp. 72-88). Paris : Retz.

Gombert, J.-E. (1990). Le développement métalinguistique. Paris : PUF.

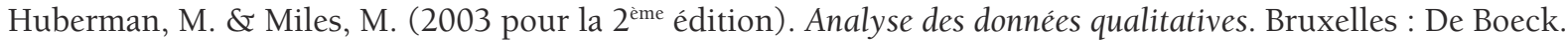

K.u.U. (1994). Bildungsplan für die Grundschule. Stuttgart: Ministerium für Kultus, Jugend und Sport Baden-Württemberg.

K.u.U. (1997). Lese- und Rechtschreibprobleme in der Grundschule. Stuttgart : Landes Institut für Erziehung und Unterricht.

K.u.U. (1999). LRS. Stuttgart : Ministerium für Kultus, Jugend und Sport Baden-Württemberg.

MEN. (1999). Etude spécifique relative aux élèves en difficulté en lecture à l'entrée en $6^{\text {ème }}$. Paris : MEN.

MEN. (2002). Littérature. Document d'application des programmes. Paris : CNDP.

MEN. (2003). Lire au CP. Paris: Ministère de l'Education nationale, de l'Enseignement supérieur et de la Recherche.

MEN. (2004). Lire au CP (2). Paris: Ministère de l'Education nationale, de l'Enseignement supérieur et de la Recherche.

MEQ. (2001). Programme de formation de l'école québécoise: Education préscolaire, enseignement primaire. Québec: Gouvernement du Québec. Ministère de l'Education.

MEQ. (2003). Les difficultés d'apprentissage à l'école. Cadre de référence pour guider l'intervention. Québec: Gouvernement du Québec. Ministère de l'éducation.

Michel, H.-J. (Ed.). (2000). FRESCH. Grundlagen, Diagnosemöglichkeiten, praktische Übungen zum Thema LRS. Lichtenau : AOL Verlag.

Rémond, M. (2007). Que nous apprend PIRLS sur la compréhension des élèves français de 10 ans? Repères, 35, 53-68.

Rinderle, B. (2000). Übungen und Strategien für LRS Kinder. Lichtenau : AOL Verlag.

Stanovich, K. (1980). Toward an Interactive Compensatory Model of Individual Differences in the Development of Reading Fluency. Research Quarterly, 16, 32-71.

Tacke, G. (1998). Lese- Rechtschreibschwäche, Diagnose, Ursachen, Fördermöglichkeiten. LEU. Materialen Grundschule GS3. Stuttgart : Landes Institut für Erziehung und Unterricht.

Tardif, J. (1992). Pour un enseignement stratégique. Montréal : Editions Logiques.

Tauveron, C. (Ed.). (2002). Lire la littérature à l'école. Pourquoi et comment conduire cet apprentissage spécifique. De la GS au CM. Paris : Hatier.

Van Grunderbeeck, N. (1994). Les difficultés en lecture. Diagnostic et pistes d'intervention. Boucherville : Gaëtan Morin.

Van Hout, A. \& Estienne, F. (2001). Les dyslexies. Paris : Masson.

Viriot-Goeldel, C. (2006). Aider l'apprenti lecteur en difficulté à l'école primaire : une perspective comparée. Essai d'analyse théorique et praxéologique des processus d'aide dans les classes de l'enseignement primaire en France, au Québec et dans le Bade-Wurtemberg. Thèse de doctorat, Université Lyon 2.

Wimmer, H. (1993). L'acquisition de la lecture dans une orthographe plus régulière que celle de l'anglais : points de divergence. In J.-P. Jaffre, L. Sprenger-Charolles et M. Fayol (Eds.), Les actes de la Villette, (pp. 97-106). Paris: Nathan pédagogie 
AIDER L'APPRENTI-LECTEUR EN DIFFICULTÉS: ANALYSE COMPARÉE DES APPROCHES DIDACTIQUES...

Caroline Viriot-Goeldel 